\title{
Finding the Most Preferred Decision-Making Unit in Data Envelopment Analysis
}

\author{
Shirin Mohammadi, ${ }^{1}$ S. Morteza Mirdehghan, ${ }^{2}$ and Gholamreza Jahanshahloo' \\ ${ }^{1}$ Department of Mathematics, Islamic Azad University, Science and Research Branch, Tehran, Iran \\ ${ }^{2}$ Department of Mathematics, College of Sciences, Shiraz University, Shiraz, Iran \\ Correspondence should be addressed to S. Morteza Mirdehghan; mirdehghan@shirazu.ac.ir
}

Received 20 June 2016; Revised 25 October 2016; Accepted 20 November 2016

Academic Editor: Konstantina Skouri

Copyright (c) 2016 Shirin Mohammadi et al. This is an open access article distributed under the Creative Commons Attribution License, which permits unrestricted use, distribution, and reproduction in any medium, provided the original work is properly cited.

\begin{abstract}
Data envelopment analysis (DEA) evaluates the efficiency of the transformation of a decision-making unit's (DMU's) inputs into its outputs. Finding the benchmarks of a DMU is one of the important purposes of DEA. The benchmarks of a DMU in DEA are obtained by solving some linear programming models. Currently, the obtained benchmarks are just found by using the information of the data of inputs and outputs without considering the decision-maker's preferences. If the preferences of the decision-maker are available, it is very important to obtain the most preferred DMU as a benchmark of the under-assessment DMU. In this regard, we present an algorithm to find the most preferred DMU based on the utility function of decision-maker's preferences by exploring some properties on that. The proposed method is constructed based on the projection of the gradient of the utility function on the production possibility set's frontier.
\end{abstract}

\section{Introduction}

Data envelopment analysis (DEA) is a nonparametric approach to assess the relative efficiencies of decision-making units (DMUs). Using some postulates in DEA, a set of possible DMUs is constructed which is called the production possibility set (PPS). One of the well-known postulates to construct the PPS is convexity assumption. If this is done, then the PPS is a convex set and its frontier is considered as an estimation of the production function. The frontier of the PPS is a collection of possible (virtual) DMUs which are weakly efficient, although some of them are strongly efficient. In other words, the frontier of the PPS is divided into two sets: strong efficient frontier and weak efficient frontier. The other DMUs in the PPS which are not on the frontier are inefficient. DEA obtains both efficiency scores and reference (benchmark) units for inefficient DMUs. The reference set for an inefficient DMU consists of all observed efficient DMUs on the efficient surface which the projection of the inefficient DMU lies on. In DEA the reference units are found by projecting a DMU on the efficient frontier.
Several models have been presented to find the reference sets in DEA. Thanassoulis and Dyson [1] have proposed a model which is based on the improvements of the weights for inputoutput levels. Zhu [2] has gathered preference information from decision-maker and then has obtained target by means of these pieces information. Also, Jahanshahloo et al. [3] and Mehdiloozad et al. [4] have proposed two approaches to find the reference set of an inefficient DMU.

DEA is a linear programming problem technique to investigate the efficiency status of DMUs. In this regard, some researchers have shown that most of the DEA models are transferred to the multiobjective linear programming (MOLP) models. In other words, the efficient DMUs can be obtained by using an MOLP problem. DEA and MOLP are helpful for managements to draw a plan for future. DEA is used to evaluate the past preferences while MOLP is used for future preferences. The similarities and relations between DEA and MOLP have been discussed by some researchers. Belton and Vickers [5] and Stewart [6] explained the equivalency between DEA and multiple criteria decision analysis (MCDA) formulations. Belton and Stewart [7] found 
that DEA emphasizes evaluating DMUs and finding targets to improve efficiency while MCDA focuses on ranking based on a set of criteria that include subjective judgment. DEA models do not involve DMU's preferences to find the efficiency of DMUs. In basic DEA models, the decision-maker's (DM's) preferences are not considered to find the benchmark of a DMU. Because of the importance of considering the DM's preferences, several methods have been proposed to incorporate the DM's preferences in DEA. Golany [8], Thanassoulis and Dyson [1], Thompson et al. [9], Wong and Beasley [10], and Zhu [2] considered prior preferences of the DM. On the other hand, the MOLP interactive techniques incorporate preference information without requiring prior judgment. Post and Spronk [11], Golany [8], and Joro et al. [12] have been talking about this concept. Wong et al. [13] obtained the relationship between the output-oriented DEA models and min-max formulation. Ebrahimnejad and Hosseinzadeh Lotfi [14] established the equivalence relationship between input- and output-oriented CCR model and the weighted min-max MOLP formulation. Yang and $\mathrm{Xu}$ [15] investigated the relationships between DEA and multiobjective optimization models for equivalent efficiency analysis in a multiobjective optimization process.

As discussed above, finding the reference of an inefficient DMU is one of the important purposes of DEA. In Golany [8], some of hypothetical or virtual units are obtained and one of them is used as a reference unit. However, some techniques require preference knowledge of decision-makers from the beginning of their techniques such as Wierzbicki [16], Zhu [2], and Thompson et al. [9]. Considering the DM's preferences is one of the methods to find the reference of a DMU which may be used when the DM's preferences existed. Yang and Sen [17], Yang [18], and Yang and Li [19] proposed an interactive procedure based on gradient projection to find a most preferred solution (MPS) in multiobjective optimization. MPS can maximize the DM's preferences. Yang [18] established his paper to present an algorithm to obtain MPS in MOLP problems. Min-max reference point approach and its application for multiobjective problems were established by Yang [20]. Yang et al. [21] used three equivalence models in MOLP, including the super-ideal point model, the ideal point model, and the shortest distance model. These models can be used for setting target values by individuals for groups. Their model can be used to find a normal vector at an efficient solution on the efficient frontier. A normal vector provides information about indifference tradeoff. On the other hand the projection of the gradient of the utility function on the efficient frontier shows a direction to move along a better efficient solution. They used the shortest distance model to find the locally most preferred solution. This point can be considered as a reference with both group and individual DM's preferences.

In this paper, we would like to find the most preferred DMU in the PPS where the utility function of DM's preferences is available. The method is constructed based on the DEA concepts and models using some MOLP principles. In the proposed method, the gradient vector of the utility function at an efficient DMU is projected on the efficient frontier and, by finding a suitable direction on the frontier, a new efficient DMU is obtained with a better utility function value. This paper is organized as follows. In Section 2 some of DEA backgrounds are reviewed. The properties and theories to find the preferred efficient DMU using the gradient projection of the utility function and the normal vectors of the PPS are established in Section 3. An algorithm to find the most preferred DMU is proposed in Section 4 using the utility function and the normal vector of the PPS which is obtained from the multiplier BCC model. A conclusion for research is summarized in Section 5.

\section{Background of DEA}

In this section some fundamental definitions, concepts, and properties in DEA are presented. Suppose that we have $n$ DMUs with $m$ inputs and $s$ outputs. The input and output vectors of $\mathrm{DMU}_{j}(j=1, \ldots, n)$ are presented by $X_{j}=\left(x_{1 j}, \ldots\right.$, $\left.x_{m j}\right)$ and $Y_{j}=\left(y_{1 j}, \ldots, y_{s j}\right)$, respectively. The production possibility set used in this paper is constructed by variable returns to scale, convexity, and possibility (free disposal hull) postulates. This PPS, which is denoted by $T_{v}$, is mathematically clarified as follows:

$$
\begin{aligned}
T_{v} & =\left\{(X, Y) \mid X \geq \sum_{j=1}^{n} \lambda_{j} X_{j}, Y \leq \sum_{j=1}^{n} \lambda_{j} Y_{j}, \sum_{j=1}^{n} \lambda_{j}\right. \\
& \left.=1, \lambda_{j} \geq 0, j=1,2, \ldots, n\right\} .
\end{aligned}
$$

Definition 1. $\mathrm{DMU}_{o}$ is efficient if there does not exist any member of the PPS which dominates $\mathrm{DMU}_{o}$. In other words, there does not exist any $(X, Y) \neq\left(X_{o}, Y_{o}\right)$ in $T_{v}$ (PPS) such that $X \leq X_{o}$ and $Y \geq Y_{o}$.

There are several DEA models which evaluate DMUs in different point of views. These models generate efficient DMUs by projecting the DMU on the efficient frontier. The criteria of this projection depend on the decision-maker's perspective. To evaluate $\mathrm{DMU}_{p}(p \in\{1,2, \ldots, n\})$, the following model, which is called the input-oriented BCC model, can be used:

$$
\begin{array}{ll}
\min & \theta \\
\text { s.t } & \sum_{j=1}^{n} \lambda_{j} X_{j} \leq \theta X_{p}, \\
& \sum_{j=1}^{n} \lambda_{j} Y_{j} \geq Y_{p}, \\
& \sum_{j=1}^{n} \lambda_{j}=1, \\
& \lambda_{j} \geq 0, \quad j=1,2, \ldots, n .
\end{array}
$$

In this model $\theta^{*}$ is the efficiency score of $\mathrm{DMU}_{p}$, where “*” indicates the optimality of (2). If $\theta^{*}=1$, then $\mathrm{DMU}_{p}$ is a 
weakly efficient DMU. Furthermore, if all constraints (except nonnegativity inequalities) are binding at optimality, then $\mathrm{DMU}_{p}$ is efficient. If $\theta^{*}<1$, then $\mathrm{DMU}_{p}$ is not an efficient DMU and $\theta^{*}$ shows the amount of the contraction of $\mathrm{DMU}_{p}$ 's inputs that it becomes efficient. Let $S E$ be the set of all efficient DMUs. For inefficient $\mathrm{DMU}_{p}$, the reference set is shown by

$$
\begin{aligned}
E_{p} & =\left\{\mathrm{DMU}_{j} \in S E \mid \lambda_{j}^{*}>0, j\right. \\
& =1,2, \ldots, n, \text { in some optimal solutions of }(2)\} .
\end{aligned}
$$

The virtual DMU $\left(\sum_{j=1}^{n} \lambda_{j}^{*} X_{j}, \sum_{j=1}^{n} \lambda_{j}^{*} Y_{j}\right)$ which is on the efficient frontier is called the projection of $\mathrm{DMU}_{p}$ on the efficient frontier and can be considered as a target for $\mathrm{DMU}_{p}$. Note that in this target the DM's preferences have not been considered. The dual of (2) which is called the input-oriented multiplier BCC model is as follows:

$$
\begin{aligned}
\max & U Y_{p}+u_{0} \\
\text { s.t. } & V X_{P}=1, \\
& U Y_{j}-V X_{j}+u_{0} \leq 0, \quad j=1,2, \ldots, n, \\
& U \geq 0, V \geq 0,
\end{aligned}
$$

where $U=\left(u_{1}, \ldots, u_{s}\right)$ and $V=\left(v_{1}, \ldots, v_{m}\right)$ are $s$ and $m$ vectors in $R_{+}^{s}$ and $R_{+}^{m}$, respectively. $\mathrm{DMU}_{p}$ is efficient if there exists an optimal solution of $(4)$ such as $\left(U^{*}, V^{*}, u_{0}^{*}\right)$ such that $U^{*} Y_{P}+u_{0}^{*}=1$ and $\left(U^{*}, V^{*}\right)>0$.

Definition 2. $H_{p}: U^{*} Y-V^{*} X+u_{0}^{*}=0$ is a supporting hyperplane of the PPS if the following conditions hold:

(1) $H_{p} \cap$ PPS $\neq \emptyset$.

(2) $\forall(X, Y)\left((X, Y) \in\right.$ PPS $\left.\rightarrow U^{*} Y-V^{*} X+u_{0}^{*} \leq 0\right)$.

Definition 3. $H$ is an efficient defining hyperplane of the PPS if it is a supporting hyperplane of the PPS, and there exists at least one affine independent set with $m+s$ elements of efficient DMUs that lie on $H$.

Theorem 4. If $\left(U^{*}, V^{*}, u_{0}^{*}\right)$ is an optimal solution of (4), then $H_{p}: U^{*} Y-V^{*} X+u_{0}^{*}=0$ is a supporting hyperplane on the PPS.

Proof. See Cooper et al. [22].

Usually, the multiplier BCC model (4) has alternative optimal solutions. Each optimal solution of (4) yields a supporting hyperplane on the PPS. If the optimal solution of (4) is unique, then the corresponding supporting hyperplane is a defining hyperplane and if (4) has alternative optimal solutions, then there are uncountable supporting hyperplanes on the PPS at the projection of $\mathrm{DMU}_{p}$ on the efficient frontier in which a finite number of them are defining hyperplanes. Jahanshahloo et al. [23] show that, in evaluating an efficient DMU, each extreme optimal solution of (4) such that $\left(U^{*}, V^{*}\right)>0$ identifies an efficient defining hyperplane of the PPS.
Theorem 5. Suppose that $D M U_{p}$ is efficient. $H_{p}: U^{*} Y-$ $V^{*} X+u_{0}^{*}=0$ is an efficient defining hyperplane of the PPS $\left(T_{v}\right)$ if and only if $\left(U^{*}, V^{*}, u_{0}^{*}\right)$ is an extreme (basic feasible) optimal solution of $(4)$ in evaluating $D M U_{p}$ with $\left(U^{*}, V^{*}\right)>0$.

Proof. see Jahanshahloo et al. [23].

Definition 6. The point that has the minimum value among the inputs and the maximum value among the outputs of all DMUs is called the ideal point and is shown by $\left(X^{I}, Y^{I}\right)$. In other words, if $x_{i}^{I}=\min \left\{x_{i} \mid\left(x_{1}, \ldots, x_{m}, y_{1}, \ldots, y_{s}\right) \in\right.$ PPS $\}$ and $y_{r}^{I}=\max \left\{y_{r} \mid\left(x_{1}, \ldots, x_{m}, y_{1}, \ldots, y_{s}\right) \in\right.$ PPS $\}$ for all $i=$ $1, \ldots, m$ and $r=1, \ldots, s$, then $\left(X^{I}, Y^{I}\right)=\left(x_{1}^{I}, \ldots, x_{m}^{I}, y_{1}^{I}, \ldots\right.$, $\left.y_{s}^{I}\right)$ is an ideal point. Usually the ideal point is not in the PPS.

\section{Finding a Preferred Efficient DMU Using the Gradient Projection of the Utility Function}

In this section, we would like to find a target of DMUs in the PPS which has the best utility function value when the utility function of the DM's preferences is available. This target is called the most preferred DMU. In this regard, we assume that $u(X, Y)$ is the utility function of the DM's preferences. In the remaining of this paper, we use the lower index for an observed DMU such as $\mathrm{DMU}_{j}$ and the upper index for a virtual DMU which, possibly, is unobserved such as $\mathrm{DMU}^{k}$. $\mathrm{DMU}^{k}$ with activity vector $\left(X^{k}, Y^{k}\right)$ clarifies the generated DMU in the $k$ th iteration of the algorithm.

Let $\mathrm{DMU}^{k-1}$ be an efficient DMU in the PPS. To move to another efficient DMU in the PPS which is shown by $\mathrm{DMU}^{k}$ we use the following linear programming problem:

$$
\begin{array}{ll}
\max & \sum_{i=1}^{m} \delta_{i} q_{i}+\sum_{r=1}^{s} \gamma_{r} p_{r} \\
\text { s.t. } & \left(X^{k}, Y^{\mathrm{k}}, Q, P\right) \in \Lambda,
\end{array}
$$

where $\left(X^{k}, Y^{k}, Q, P\right)=\left(x_{1}^{k}, \ldots, x_{m}^{k}, y_{1}^{k}, \ldots, y_{s}^{k}, q_{1}, \ldots, q_{m}, p_{1}\right.$, $\left.\ldots, p_{s}\right)$ and

$$
\begin{aligned}
\Lambda & =\left\{\left(X^{k}, Y^{k}, Q, P\right) \mid x_{i}^{k} \leq x_{i}^{k-1}+\Delta x_{i}^{k-1}-q_{i}, y_{r}^{k}\right. \\
& \geq y_{r}^{k-1}-\Delta y_{r}^{k-1}+p_{r}, q_{i} \geq 0, p_{r} \geq 0,\left(X^{k}, Y^{\mathrm{k}}\right) \\
& \in \mathrm{PPS}, i=1, \ldots, m, \quad r=1, \ldots, s\} .
\end{aligned}
$$

In the above model at first the DMU throws in the PPS by decreasing $\Delta Y$ from outputs and adding $\Delta X$ to the inputs of $\mathrm{DMU}^{k-1}$. Then, it arises on the efficient frontier by adding $P$ to outputs and subtracting $Q$ from inputs. In problem (5), $\Delta y_{r}^{k-1}, \Delta x_{i}^{k-1}, \gamma_{r}$, and $\delta_{i}$ are nonnegative constants and $p_{r}$ and $q_{i}$ are the auxiliary variables for all $i=1,2, \ldots, m$ and $r=$ $1,2, \ldots, s$.

To reach a DMU which has the better utility function value than the current efficient DMU, it is sufficient to project the gradient of the utility function on the efficient frontier 
at the current DMU, because the utility function value is increased at the ascent direction of the gradient projection.

Remark 7. To find a DMU which is better than $\left(X^{k-1}, Y^{k-1}\right)$ in the base of the utility function value, $\Delta y_{r}^{k-1}, \Delta x_{i}^{k-1}, \gamma_{r}$, and $\delta_{i}$ should be determined at stage $k-1$. These scalars are determined based on the gradient of the utility function and its projection on the efficient frontier at $\left(X^{k-1}, Y^{k-1}\right)$.

Theorem 8. Suppose that $\left(X^{k^{*}}, Y^{k^{*}}, Q^{*}, P^{*}\right)$ is an optimal solution of (5).

(1) If $\delta_{i}>0$ and $\gamma_{r}>0$, where $i=1,2, \ldots, m$ and $r=$ $1,2, \ldots, s$, then $\left(X^{k^{*}}, Y^{k^{*}}\right)$ is an efficient DMU in the PPS.

(2) Generally, if $(\delta, \gamma)=\left(\delta_{1}, \ldots, \delta_{m}, \gamma_{1}, \ldots, \gamma_{s}\right) \geq 0$ and $(\delta, \gamma) \neq 0$, then $\left(X^{k^{*}}, Y^{k^{*}}\right)$ is a weakly efficient DMU in the PPS.

Proof. Let $\delta_{i}>0$ and $\gamma_{r}>0$ for all $i=1,2, \ldots, m$ and $r=$ $1,2, \ldots, s$. Moreover, consider $\left(x_{i}^{k-1}+\Delta x_{i}^{k-1}, y_{r}^{k-1}-\Delta y_{r}^{k-1}\right) \epsilon$ PPS, so problem (5) is rewritten as follows:

$$
\begin{aligned}
& \max \sum_{i=1}^{m} \delta_{i} q_{i}+\sum_{r=1}^{s} \gamma_{r} p_{r} \\
& \text { s.t } \quad x_{i}^{k} \leq x_{i}^{k-1}+\Delta x_{i}^{k-1}-q_{i}, \quad i=1,2, \ldots, m, \\
& y_{r}^{k} \geq y_{r}^{k-1}-\Delta y_{r}^{k-1}+p_{r}, \quad r=1,2, \ldots, s, \\
& x_{i}^{k} \geq \sum_{j=1}^{n} \lambda_{j} x_{i j}, \quad i=1,2, \ldots, m, \\
& y_{r}^{k} \leq \sum_{j=1}^{n} \lambda_{j} y_{r j}, \quad r=1,2, \ldots, s, \\
& p_{r} \geq 0, \quad r=1,2, \ldots, s, \\
& q_{i} \geq 0, \quad i=1,2, \ldots, m,
\end{aligned}
$$

where $x_{i}^{k}, y_{r}^{k}, q_{i}$, and $p_{r}, i=1,2, \ldots, m$ and $r=1,2, \ldots, s$, are variables. Let $\left(X^{k^{*}}, Y^{k^{*}}, Q^{*}, P^{*}\right)$ be the optimal solution of problem (7). So we have

$$
\begin{aligned}
& x_{i}^{k^{*}}=x_{i}^{k-1}+\Delta x_{i}^{k-1}-q_{i}^{*}, \quad i=1,2, \ldots, m, \\
& y_{r}^{k^{*}}=y_{r}^{k-1}-\Delta y_{r}^{k-1}+p_{r}^{*}, \quad r=1,2, \ldots, s .
\end{aligned}
$$

By contradictions, suppose that $\left(X^{k^{*}}, Y^{k^{*}}\right)$ is not an efficient DMU and another DMU such as $(\bar{X}, \bar{Y})$ is an efficient DMU in the PPS which dominates $\left(X^{k^{*}}, Y^{k^{*}}\right)$. Therefore, $\bar{X} \leq X^{k^{*}}, \bar{Y} \geq Y^{k^{*}}$ and at least one inequality is strict. This implies that there exist vectors $T=\left(t_{1}, t_{2}, \ldots, t_{m}\right) \in R_{+}^{m}$ and
$N=\left(n_{1}, n_{2}, \ldots, n_{s}\right) \in R_{+}^{s}$ such that $\bar{X}+T=X^{k^{*}}, \bar{Y}-N=Y^{k^{*}}$, and $(T, N) \neq 0$. So from (8) we have

$$
\begin{aligned}
& \overline{x_{i}}+t_{i}=x_{i}^{k-1}+\Delta x_{i}^{k-1}-q_{i}^{*}, \quad i=1,2, \ldots, m, \\
& \overline{y_{r}}-n_{r}=y_{r}^{k-1}-\Delta y_{r}^{k-1}+p_{r}^{*}, \quad r=1,2, \ldots, s, \\
& (T, N) \geq 0, \\
& (T, N) \neq 0 .
\end{aligned}
$$

Let $\widetilde{y_{r}}=\overline{y_{r}}, \widetilde{x_{i}}=\overline{x_{i}}, \widetilde{p_{r}}=p_{r}^{*}+n_{r}$, and $\widetilde{q_{i}}=q_{i}^{*}+t_{i}$ for $i=1,2, \ldots, m$ and $r=1,2, \ldots, s$. Therefore, $(\widetilde{X}, \widetilde{Y}, \widetilde{Q}, \widetilde{P})$ is a feasible solution for problem $(7)$ such that $(\widetilde{Q}, \widetilde{P}) \geq\left(Q^{*}, P^{*}\right)$ and $(\widetilde{Q}, \widetilde{P}) \neq\left(Q^{*}, P^{*}\right)$. Because of $\gamma_{r}>0$ and $\delta_{i}>0$ for all $i$ and $r$, the objective function's value of problem (7) corresponding to this feasible solution, $(\widetilde{X}, \widetilde{Y}, \widetilde{Q}, \widetilde{P})$, is larger than the optimal value of (7) and this contradicts with our assumption that $\left(X^{k^{*}}, Y^{k^{*}}, Q^{*}, P^{*}\right)$ is an optimal solution of (5) and (7). erty 1 .

The proof of property 2 is similar to the proof of prop-

Theorem 9. Let $\left(X^{k}, Y^{k}\right) \in S E$ and $\left(X^{I}, Y^{I}\right)$ be the ideal point. Suppose that $w_{r}=1 /\left(y_{r}^{I}-y_{r}^{k}\right)(r=1,2, \ldots, s)$ and $z_{i}=1 /\left(x_{i}^{k}-\right.$ $\left.x_{i}^{I}\right)(i=1,2, \ldots, m)$. If $y_{r}^{I}-y_{r}^{k}>0$ and $x_{i}^{k}-x_{i}^{I}>0$ for all $i$ and $r$, then $\left(X^{k}, Y^{k}\right)$ is an optimal solution of the following problem:

$$
\min _{(X, Y) \in \operatorname{PPS}} \max _{r, i}\left\{w_{r}\left(y_{r}^{I}-y_{r}\right), z_{i}\left(x_{i}-x_{i}^{I}\right)\right\} .
$$

Proof. Problem (10) is equivalent to the following problem:

$$
\begin{array}{ll}
\min & \theta \\
\text { s.t. } & w_{r}\left(y_{r}^{I}-y_{r}\right) \leq \theta, \quad r=1,2, \ldots, s, \\
& z_{i}\left(x_{i}-x_{i}^{I}\right) \leq \theta, \quad i=1,2, \ldots, m, \\
& (X, Y) \in \mathrm{PPS} \\
& \theta \geq 0
\end{array}
$$

where $x_{i}, y_{r}$, and $\theta, i=1,2, \ldots, m$ and $r=1,2, \ldots, s$, are variables.

By contradiction, suppose that $\left(X^{k}, Y^{k}\right)$ is not an optimal solution of this problem. Therefore, there exists $(\bar{X}, \bar{Y})$ such that $(\bar{X}, \bar{Y})$ is an optimal solution of (11) and $\bar{\theta}$ is the optimal value of that. We have $\bar{\theta}<1$ because $\left(X^{k}, Y^{k}, \theta^{k}\right)$, where $\theta^{k}=$ 1 , is a feasible solution of problem (11). Moreover, $w_{r}\left(y_{r}^{I}-\right.$ $\left.\overline{y_{r}}\right) \leq \bar{\theta}<\theta^{k}=1$ and then $w_{r}\left(y_{r}^{I}-\overline{y_{r}}\right)<1$. By the assumption of $w_{r}>0$ and the definition of the ideal point, we have

$$
y_{r}^{k}<\overline{y_{r}}, \quad r=1,2, \ldots, s .
$$

Similarly, $z_{i}\left(\overline{x_{i}}-x_{i}^{I}\right) \leq \bar{\theta}<\theta^{k}=1$ and we can conclude that

$$
x_{i}^{k}>\overline{x_{i}}, \quad i=1,2, \ldots, m .
$$

(A) and (B) imply that $\left(X^{k}, Y^{k}\right)$ is dominated by $(\bar{X}, \bar{Y})$ and this contradicts with the assumption that $\left(X^{k}, Y^{k}\right)$ is efficient. 
Therefore, $\left(X^{k}, Y^{k}\right)$ is the optimal solution of problems (10) and (11).

Suppose that $N^{k}$ is a unique normal vector of the PPS at $\left(X^{k}, Y^{k}\right)$. Because the PPS is a polyhedron, $N^{k}$ is the normal vector of a defining hyperplane of the PPS at $\left(X^{k}, Y^{k}\right)$. Using $N^{k}$, we can find the projection of the gradient of the utility function on the efficient frontier at this point. In other words, if $\nabla u\left(X^{k}, Y^{k}\right)$ is not orthogonal to any tangent plane (defining hyperplane) of the PPS at $\left(X^{k}, Y^{k}\right)$, then we can project $\nabla u\left(X^{k}, Y^{k}\right)$ on the tangent plane of the PPS at this point. This projection provides a direction at $\left(X^{k}, Y^{k}\right)$ in which the utility function value can be increased.

Remark 10. To obtain a defining hyperplane of the PPS at an efficient DMU, it is sufficient to solve problem (4) corresponding to the mentioned DMU. So if $\left(U^{*}, V^{*}, u_{0}^{*}\right)$, such that $\left(U^{*}, V^{*}\right)>0$, is an extreme optimal solution of $(4)$, then $U^{*} Y-V^{*} X+u_{0}^{*}=0$ is a defining hyperplane of the PPS and $\left(U^{*},-V^{*}\right)$ is the normal vector of this hyperplane (see Jahanshahloo et al. [23]).

Theorem 11. Let $\left(X^{k}, Y^{k}\right) \in S E$ and the gradient of the utility function at $\left(X^{k}, Y^{k}\right)$ is given by $\nabla u\left(X^{k}, Y^{k}\right)=\left(\partial u\left(X^{k}, Y^{k}\right) /\right.$ $\partial x_{1}, \ldots, \partial u\left(X^{k}, Y^{k}\right) / \partial x_{m}, \partial u\left(X^{k}, Y^{k}\right) / \partial y_{1}, \ldots, \partial u\left(X^{k}, Y^{k}\right) /$

$\left.\partial y_{s}\right)$. If $\left(X^{k}, Y^{k}\right)$ is a member of the PPS which has the best utility function value, then there exists a supporting hyperplane of the PPS at $\left(X^{k}, Y^{k}\right)$ whose normal vector is parallel to the gradient vector of the utility function at $\left(X^{k}, Y^{k}\right)$; that is,

$$
\nabla u\left(X^{k}, Y^{k}\right)=c N^{k}
$$

where $N^{k}$ is the normal vector of a supporting hyperplane on the PPS at $\left(X^{k}, Y^{k}\right)$ and $c$ is a positive scalar number.

Proof. When $\left(X^{k}, Y^{k}\right)$ has the best utility function value, we can conclude that there does not exist any ascent direction of $u$ on the efficient frontier at $\left(X^{k}, Y^{k}\right)$ such that we can move from $\left(X^{k}, Y^{k}\right)$ on the efficient frontier and the value of $u$ is increased. Because of the convexity of the PPS, we have $\nabla u\left(X^{k}, Y^{k}\right) \cdot\left(X-X^{k}, Y-Y^{k}\right) \leq 0$; that is, $\nabla u\left(X^{k}, Y^{k}\right) \cdot(X, Y) \leq$ $\nabla u\left(X^{k}, Y^{k}\right) \cdot\left(X^{k}, Y^{k}\right)$ for all $(X, Y) \in$ PPS. This implies that $H=\left\{(X, Y) \in R^{m+s} \mid \nabla u\left(X^{k}, Y^{k}\right) \cdot(X, Y)=t\right\}$ is a supporting hyperplane of the PPS binding at $\left(X^{k}, Y^{k}\right)$ where $t=\nabla u\left(X^{k}, Y^{k}\right) \cdot\left(X^{k}, Y^{k}\right)$.

Result. Let $N^{1}, \ldots, N^{v}$ be the normal vectors of the defining hyperplanes of the PPS binding at $\left(X^{k}, Y^{k}\right)$. If $\nabla u\left(X^{k}, Y^{k}\right) \in$ $\operatorname{Pos}\left\{N^{1}, \ldots, N^{v}\right\}$, then $\left(X^{k}, Y^{k}\right)$ is a most preferred DMU, where $\operatorname{Pos}\left\{N^{1}, \ldots, N^{v}\right\}=\left\{N \mid N=\sum_{j=1}^{v} \alpha_{j} N^{j}, \alpha_{j} \geq 0, j=\right.$ $1,2, \ldots, v\}$.

Remark 12. If $\left(X^{k}, Y^{k}\right)$ is a relative interior point of a facet of the PPS, then the normal vector of the PPS at $\left(X^{k}, Y^{k}\right)$ is unique.
Condition (12) shows that if $\left(X^{k}, Y^{k}\right)$ is an efficient DMU with the best utility function value, then the projection of the gradient of $u$ at $\left(X^{k}, Y^{k}\right)$ on the efficient frontier is zero. This shows that there does not exist any ascent direction of $u$ at $\left(X^{k}, Y^{k}\right)$ and so $\left(X^{k}, Y^{k}\right)$ is the most preferred DMU. Otherwise, if $\left(X^{k}, Y^{k}\right)$ is not a most preferred DMU, then we project the gradient of $u$ on the efficient frontier. This projection shows the ascent direction of $u$ at $\left(X^{k}, Y^{k}\right)$. In this regard, suppose that $N^{k}$ is the unique normal vector of the PPS at $\left(X^{k}, Y^{k}\right)$ and $\theta$ is the angle between $\nabla u\left(X^{k}, Y^{k}\right)$ and $N^{k}$. If $\theta>0$, then the tangent plane of the PPS, which is orthogonal to $N^{k}$, is not parallel to a plane which is orthogonal to $\nabla u\left(X^{k}, Y^{k}\right)$. So we can proceed from $\left(X^{k}, Y^{k}\right)$ at the ascent direction of $u$. Otherwise, if $\theta=0$, then the most preferred DMU has been obtained.

Remark 13. If $\left(X^{k}, Y^{k}\right)$ is an efficient DMU and there exist more than one defining hyperplane binding at this DMU, then we have multiple normal vectors of the PPS at $\left(X^{k}, Y^{k}\right)$. In this case, we choose one of the normal vectors which has the minimum angle with $\nabla u\left(X^{k}, Y^{k}\right)$; after that, $\nabla u\left(X^{k}, Y^{k}\right)$ is projected on the defining hyperplane corresponding to the chosen normal vector.

Theorem 14. Suppose that $\left(X^{k}, Y^{k}\right)$ is an efficient DMU and $\nabla u\left(X^{k}, Y^{k}\right)$ is the gradient of $u$ at this DMU. If $d\left(X^{k}, Y^{k}\right) \neq$ 0 , denoted as follows, is the projection of $\nabla u\left(X^{k}, Y^{k}\right)$ on the defining hyperplane of the PPS binding at this point, then $d\left(X^{k}, Y^{k}\right)$ is the ascent direction of $u$ at $\left(X^{k}, Y^{k}\right)$. Furthermore,

$$
d\left(X^{k}, Y^{k}\right)=\nabla u\left(X^{k}, Y^{k}\right)-\frac{\nabla u\left(X^{k}, Y^{k}\right) N^{k}}{N^{k} N^{k}} N^{k},
$$

where $N^{k}$ is the normal vector of the defining hyperplane of the PPS binding at $\left(X^{k}, Y^{k}\right)$.

Proof. Geometrically, (13) is clear. If relation (13) is multiplied by $\nabla u\left(X^{k}, Y^{k}\right)$, then we have

$$
\begin{aligned}
\nabla u & \left(X^{k}, Y^{k}\right) \cdot d\left(X^{k}, Y^{k}\right) \\
& =\left\|\nabla u\left(X^{k}, Y^{k}\right)\right\|^{2}\left(1-\cos ^{2} \theta\right) \\
& =\left\|\nabla u\left(X^{k}, Y^{k}\right)\right\|^{2} \sin ^{2} \theta,
\end{aligned}
$$

where $\theta$ is the angle between $\nabla u\left(X^{k}, Y^{k}\right)$ and $N^{k}$. Regarding $\theta \neq 0$, we conclude that $\nabla u\left(X^{k}, Y^{k}\right) \cdot d\left(X^{k}, Y^{k}\right)>0$, because the right hand side of the above equation is positive. Therefore, $d\left(X^{k}, Y^{k}\right)$ is the ascent direction of $u$ at $\left(X^{k}, Y^{k}\right)$.

Corollary 15. If $\left(X^{k}, Y^{k}\right)$ is an efficient DMU and it has the best utility function value, then there exists a normal vector corresponding to a supporting hyperplane of the PPS at this DMU such that $d\left(X^{k}, Y^{k}\right)=0$.

Proof. Because $\left(X^{k}, Y^{k}\right)$ has the best utility function among all DMUs in the PPS, regarding Theorem 11, there exist $c>$ 
0 and a supporting hyperplane of the PPS at $\left(X^{k}, Y^{k}\right)$ with normal vector $N^{k}$ such that $\nabla u\left(X^{k}, Y^{k}\right)=c N^{k}$. Therefore, by relation (13) we have

$$
d\left(X^{k}, Y^{k}\right)=c N^{k}-\frac{c N^{k} N^{k}}{N^{k} N^{k}} N^{k}=0 .
$$

Corollary 16. Suppose that $\left(X^{k}, Y^{k}\right)$ is an efficient DMU and it has the best utility function value. Moreover, let the normal vector of the PPS at this point be $N^{k}=\left(U^{*},-V^{*}\right)$ such that $U^{*}>0$ and $V^{*}>0$; then one has

$$
\begin{aligned}
\frac{\partial u\left(X^{k}, Y^{k}\right) / \partial y_{1}}{u_{1}^{*}} & =\cdots=\frac{\partial u\left(X^{k}, Y^{k}\right) / \partial y_{s}}{u_{s}^{*}} \\
& =-\frac{\partial u\left(X^{k}, Y^{k}\right) / \partial x_{1}}{v_{1}^{*}}=\cdots \\
& =-\frac{\partial u\left(X^{k}, Y^{k}\right) / \partial x_{m}}{v_{m}^{*}}=c .
\end{aligned}
$$

The proof of Corollary 16 can be obtained directly by Theorem 11. Note that $N^{k}=\left(U^{*},-V^{*}\right)$ can be obtained easily using Theorem 4 by solving problem (4).

\section{Finding the Most Preferred DMU in DEA: An Algorithm}

Let the gradient of the utility function at $\left(X^{k-1}, Y^{k-1}\right)$ be

$$
\begin{gathered}
\nabla u\left(X^{k-1}, Y^{k-1}\right)=\left(\frac{\partial u\left(X^{k-1}, Y^{k-1}\right)}{\partial x_{1}}, \ldots,\right. \\
\frac{\partial u\left(X^{k-1}, Y^{k-1}\right)}{\partial x_{m}}, \frac{\partial u\left(X^{k-1}, Y^{k-1}\right)}{\partial y_{1}}, \ldots, \\
\left.\frac{\partial u\left(X^{k-1}, Y^{k-1}\right)}{\partial y_{s}}\right) .
\end{gathered}
$$

Consider an arbitrary index $l \in\{1,2, \ldots, s\}$ as the index of a reference output such that $w=\partial u\left(X^{k-1}, Y^{k-1}\right) / \partial y_{l}>0$, where $w=\max \left\{\partial u\left(X^{k-1}, Y^{k-1}\right) / \partial y_{r} \mid r=1,2, \ldots, s\right\}$. Let the normalized gradient vector of the utility function at $\left(X^{k-1}\right.$, $Y^{k-1}$ ) be $G^{k-1}$ which is identified as follows:

$$
G^{k-1}=\frac{\nabla u\left(X^{k-1}, Y^{k-1}\right)}{w} .
$$

The projection of $G^{k-1}$ on the efficient frontier at $\left(X^{k-1}, Y^{k-1}\right)$ is shown by $d\left(X^{k-1}, Y^{k-1}\right)$ and it is determined as follows:

$$
d\left(X^{k-1}, Y^{k-1}\right)=G^{k-1}-\frac{G^{k-1} N^{k-1}}{N^{k-1} N^{k-1}} N^{k-1} .
$$

Let $d\left(X^{k-1}, Y^{k-1}\right)=\left(d x_{1}^{k-1}, \ldots, d x_{m}^{k-1}, d y_{1}^{k-1}, \ldots, d y_{s}^{k-1}\right)$, and

$$
\begin{aligned}
\Omega^{k} & =\left\{(X, Y, Q, P) \mid x_{i} \leq x_{i}^{k-1}+\Delta x_{i}^{k-1}-q_{i}, y_{r}\right. \\
& \geq y_{r}^{k-1}-\Delta y_{r}^{k-1}+p_{r}, q_{i} \geq 0, \quad p_{r} \geq 0, \quad(X, Y) \\
& \in \text { PPS }, i=1, \ldots, m, r=1, \ldots, s\},
\end{aligned}
$$

where

$$
\begin{aligned}
\Delta x_{i}^{k-1}=\frac{\bar{\alpha}^{k-1}}{2}\left(\left|d x_{i}^{k-1}\right|+d x_{i}^{k-1}\right), & i=1,2, \ldots, m, \\
\Delta y_{r}^{k-1} & =\frac{\bar{\alpha}^{k-1}}{2}\left(\left|d y_{r}^{k-1}\right|-d y_{r}^{k-1}\right), \quad r=1,2, \ldots, s, \\
\bar{\alpha}^{k-1} & =\alpha_{2} \cdot \alpha^{k-1} .
\end{aligned}
$$

In (22), $\alpha \in[0,1]$ is a scalar and $\alpha^{k-1}$ is the largest step size to move from $\left(X^{k-1}, Y^{k-1}\right)$ in direction $d\left(X^{k-1}, Y^{k-1}\right)$ to remain in the PPS. In other words, $\alpha^{k-1}=\max \left\{\alpha \mid\left(X^{k-1}\right.\right.$, $\left.Y^{k-1}\right)+\alpha d\left(X^{k-1}, Y^{k-1}\right) \in$ PPS $\} . \alpha^{k-1}$ is obtained by solving the following linear programming problem:

$$
\begin{aligned}
\alpha^{k-1}=\max & \alpha \\
\text { s.t. } \quad & \sum_{j=1}^{n} \lambda_{j} X_{j} \leq x_{i}^{k-1}+\alpha d x_{i}^{k-1}, \\
& \\
& \sum_{j=1}^{n} \lambda_{j} Y_{j} \geq y_{r}^{k-1}+\alpha d y_{r}^{k-1}, \\
& \\
& \sum_{j=1}^{n} \lambda_{j}=1, \ldots, m, \quad r=1,2, \ldots, s, \\
& \lambda_{j} \geq 0, \quad j=1,2, \ldots, n, \\
& \alpha \geq 0 .
\end{aligned}
$$

To find a preferred solution comparing to $\left(X^{k-1}, Y^{k-1}\right)$, we solve the following linear programming model:

$$
\begin{aligned}
\max & \sum_{i=1}^{m} \delta_{i} q_{i}+\sum_{r=1}^{s} \gamma_{r} p_{r} \\
\text { s.t. } & \left(X^{k}, Y^{k}, Q, P\right) \in \Omega^{k},
\end{aligned}
$$

where the components of $\left(X^{k}, Y^{k}, Q, P\right)=\left(x_{1}^{k}, \ldots, x_{m}^{k}, y_{1}^{k}\right.$, $\left.\ldots, y_{s}^{k}, q_{1}, \ldots, q_{m}, p_{1}, \ldots, p_{s}\right)$ are the variables. Moreover, $\delta_{i}$ and $\gamma_{r}, i=1,2, \ldots, m$ and $r=1,2, \ldots, s$, are the constants.

By the above discussions the summary of the proposed algorithm to find the most preferred DMU in DEA is presented as follows.

Step 1. Let $k:=1$ and find an initial efficient $\operatorname{DMU}\left(X^{1}, Y^{1}\right)$. To find this point (DMU) we can solve one of DEA models 
such as problem (2) for evaluating an observed DMU to project it on the efficient frontier. The projected DMU can be used as an initial efficient DMU. Note that if the evaluated observed DMU is on the efficient frontier, then it can be used as an initial efficient DMU.

Step 2. Let $k:=k+1$. Find the normal vector $\left(N^{k-1}\right)$ of the PPS at $\left(X^{k-1}, Y^{k-1}\right)$ by solving problem (4). Consider $y_{l}(l \in\{1,2, \ldots, s\})$ as the reference output. Identify $G^{k-1}$ at $\left(X^{k-1}, Y^{k-1}\right)$ by (18).

Step 3. Find $d\left(X^{k-1}, Y^{k-1}\right)$ using (19). If $d\left(X^{k-1}, Y^{k-1}\right)=$ 0 , then the most preferred DMU is $\left(X^{k-1}, Y^{k-1}\right)$ and stop. Otherwise, if $d\left(X^{k-1}, Y^{k-1}\right) \neq 0$, then go to Step 4 .

Step 4. Find the step size $\alpha^{k-1}$ by solving problem (23). Moreover, let $\alpha_{2}:=1$.

Step 5. Let $\bar{\alpha}^{k-1}:=\alpha_{2} \alpha^{k-1}$, and define $\Omega^{k}$ by (20) and (21). Furthermore, solve problem (24) to obtain $\left(X^{k}, Y^{k}\right)$. If $\left(X^{k}\right.$, $\left.Y^{k}\right)=\left(X^{k-1}, Y^{k-1}\right)$, then $\left(X^{k}, Y^{k}\right)$ is the most preferred DMU and stop; otherwise, go to Step 6.

Step 6. Find $N^{k}$ and then $d\left(X^{k}, Y^{k}\right)$. If $d\left(X^{k}, Y^{k}\right)=0$, then $\left(X^{k}, Y^{\mathrm{k}}\right)$ is the most preferred DMU and stop. If $d\left(X^{k}, Y^{k}\right)$. $d\left(X^{k-1}, Y^{k-1}\right)<0$, then let $\alpha_{2}:=\alpha_{2} / 2$ and go to Step 5 . Otherwise, if $d\left(X^{k}, Y^{k}\right) \cdot d\left(X^{k-1}, Y^{k-1}\right)>0$, then let $k:=k+1$ and go to Step 4.

\section{Conclusion}

In data envelopment analysis, usually, the projection of an inefficient DMU on the efficient frontier of the production possibility set is used as a target of the DMU. This target has two characteristics: the under-assessment inefficient DMU is dominated by its target and the target is efficient. The target is obtained corresponding to the used models. In other words, the targets, usually, are different depending on using different DEA models and the amounts of inputs and outputs of the observed DMUs. Moreover, possibly, the decision-maker has some preferences to characterize the target. In this case the standard DEA models cannot find a target of a DMU which maximizes the decision-maker's preferences. In this paper, we propose an approach to find a target of DMUs which maximizes the utility function value of the decision-maker's preferences. This approach is constructed based on finding the defining hyperplanes of the production possibility set and the projection of the gradient vector of the utility function on the defining hyperplanes of the production possibility set. By this projection, an ascent direction of the utility function is obtained and another DMU which has the better value of the utility function is obtained. By an iterative algorithm, the most preferred DMU is obtained.

\section{Competing Interests}

The authors declare that there is no conflict of interests regarding the publication of this paper.

\section{References}

[1] E. Thanassoulis and R. G. Dyson, "Estimating preferred target input-output levels using data envelopment analysis," European Journal of Operational Research, vol. 56, no. 1, pp. 80-97, 1992.

[2] J. Zhu, "Data envelopment analysis with preference structure," Journal of the Operational Research Society, vol. 47, no. 1, pp. 136$150,1996$.

[3] G. R. Jahanshahloo, A. Shirzadi, and S. M. Mirdehghan, "Finding the reference set of a decision making unit," Asia-Pacific Journal of Operational Research, vol. 25, no. 4, pp. 563-573, 2008.

[4] M. Mehdiloozad, S. M. Mirdehghan, B. K. Sahoo, and I. Roshdi, "On the identification of the global reference set in data envelopment analysis," European Journal of Operational Research, vol. 245, no. 3, pp. 779-788, 2015.

[5] V. Belton and S. P. Vickers, "Demystifying DEA-a visual interactive approach based on multiple criteria analysis," Journal of the Operational Research Society, vol. 44, no. 9, pp. 883-896, 1993.

[6] T. J. Stewart, "Relationships between data envelopment analysis and multicriteria decision analysis," Journal of the Operational Research Society, vol. 47, no. 5, pp. 654-665, 1996.

[7] V. Belton and T. J. Stewart, Multiple Criteria Decision Analysis: An Integrated Approach, Kluwer Academic, 2002.

[8] B. Golany, "An interactive MOLP procedure for the extension of DEA to effectiveness analysis," Journal of the Operational Research Society, vol. 39, no. 8, pp. 725-734, 1988.

[9] R. G. Thompson, L. N. Langemeier, C.-T. Lee, E. Lee, and R. M. Thrall, "The role of multiplier bounds in efficiency analysis with application to Kansas farming," Journal of Econometrics, vol. 46, no. 1-2, pp. 93-108, 1990.

[10] Y.-H. B. Wong and J. E. Beasley, "Restricting weight flexibility in data envelopment analysis," Journal of the Operational Research Society, vol. 41, no. 9, pp. 829-835, 1990.

[11] T. Post and J. Spronk, "Performance benchmarking using interactive data envelopment analysis," European Journal of Operational Research, vol. 115, no. 3, pp. 472-487, 1999.

[12] T. Joro, P. Korhonen, and J. Wallenius, "Structural comparison of data envelopment analysis and multiple objective linear programming," Management Science, vol. 44, no. 7, pp. 962-970, 1998.

[13] B. Y. H. Wong, M. Luque, and J.-B. Yang, "Using interactive multiobjective methods to solve DEA problems with value judgements," Computers \& Operations Research, vol. 36, no. 2, pp. 623-636, 2009.

[14] A. Ebrahimnejad and F. Hosseinzadeh Lotfi, "Equivalence relationship between the general combined-oriented CCR model and the weighted minimax MOLP formulation," Journal of King Saud University-Science, vol. 24, no. 1, pp. 47-54, 2012.

[15] J.-B. Yang and D.-L. Xu, "Interactive minimax optimisation for integrated performance analysis and resource planning," Computers \& Operations Research, vol. 46, pp. 78-90, 2014.

[16] A. P. Wierzbicki, "The use of reference objectives in multi objectives optimization," in Multiple Criteria Decision Making, Theory and Application, G. Fandel and T. Gal, Eds., Springer, New York, NY, USA, 1980.

[17] J.-B. Yang and P. Sen, "Preference modelling by estimating local utility functions for multiobjective optimization," European Journal of Operational Research, vol. 95, no. 1, pp. 115-138, 1996.

[18] J.-B. Yang, "Gradient projection and local region search for multiobjective optimization," European Journal of Operational Research, vol. 112, no. 2, pp. 432-459, 1999. 
[19] J.-B. Yang and D. Li, "Normal vector identification and interactive tradeoff analysis using minimax formulation in multiobjective optimization," IEEE Transactions on Systems, Man, and Cybernetics Part A:Systems and Humans., vol. 32, no. 3, pp. 305$319,2002$.

[20] J.-B. Yang, "Minimax reference point approach and its application for multiobjective optimisation," European Journal of Operational Research, vol. 126, no. 3, pp. 541-556, 2000.

[21] J.-B. Yang, B. Y. H. Wong, D.-L. Xu, and T. J. Stewart, "Integrating DEA-oriented performance assessment and target setting using interactive MOLP methods," European Journal of Operational Research, vol. 195, no. 1, pp. 205-222, 2009.

[22] W. W. Cooper, L. M. Seiford, and K. Tone, Introduction to Data Envelopment Analysis and Its Uses: With DEA-Solver Software and References, Springer Science \& Business Media, 2006.

[23] G. R. Jahanshahloo, A. Shirzadi, and S. M. Mirdehghan, "Finding strong defining hyperplanes of PPS using multiplier form," European Journal of Operational Research, vol. 194, no. 3, pp. 933-938, 2009. 


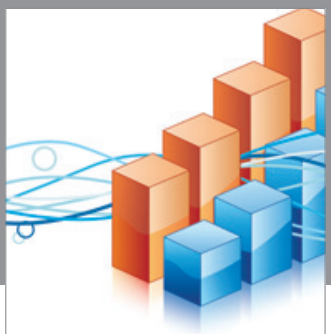

Advances in

Operations Research

vatem alat4

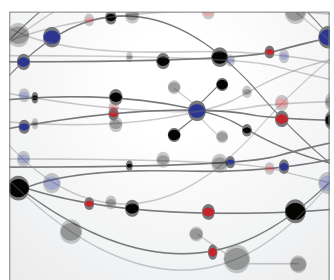

\section{The Scientific} World Journal
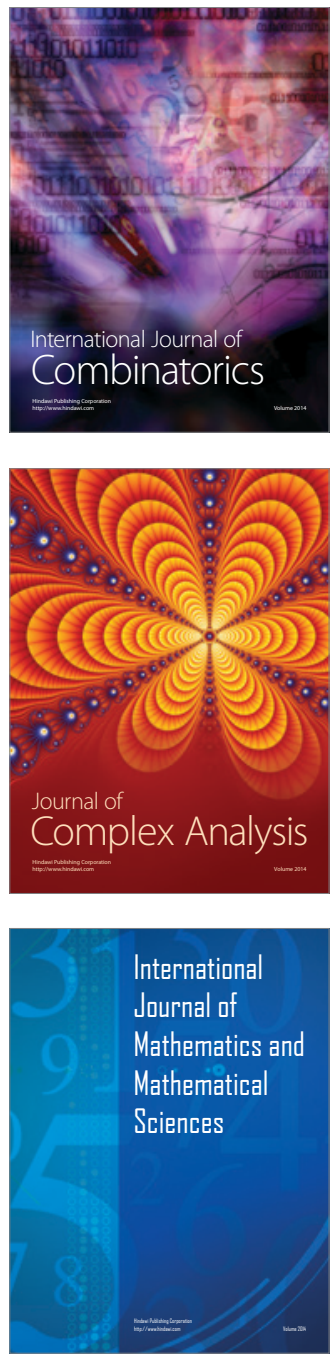
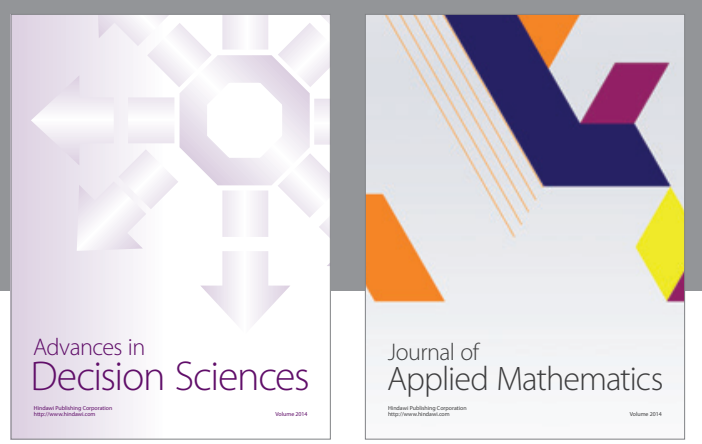

Algebra

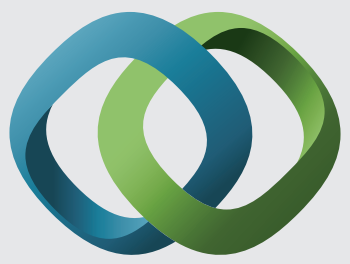

\section{Hindawi}

Submit your manuscripts at

http://www.hindawi.com
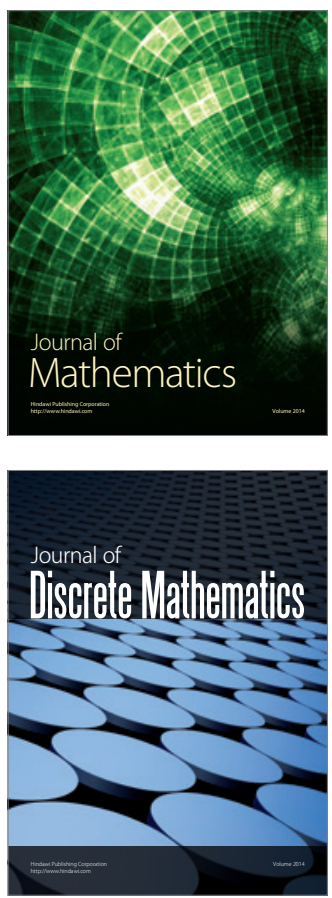

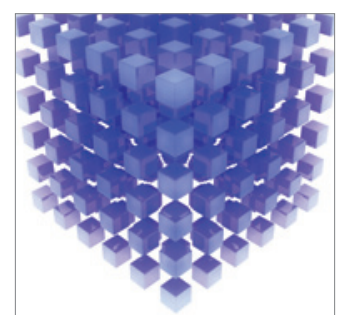

Mathematical Problems in Engineering
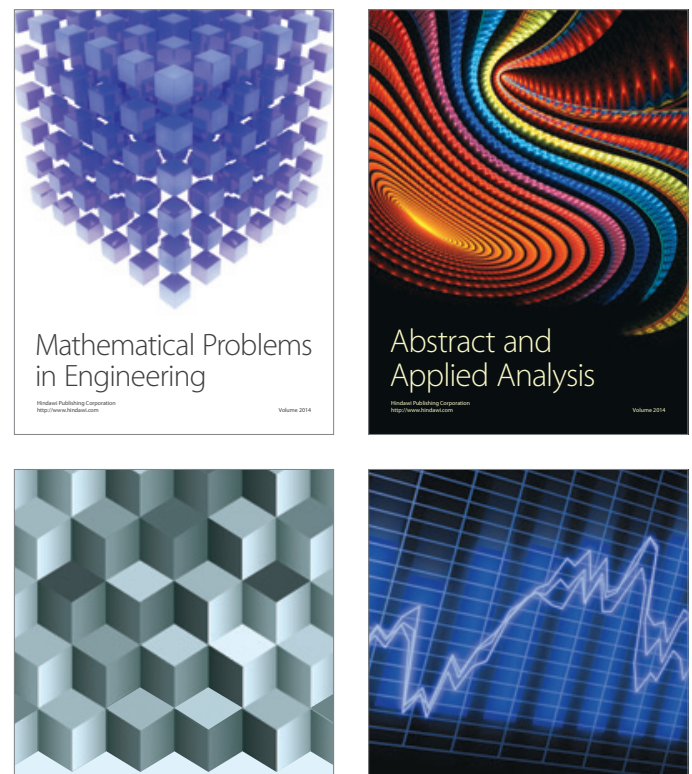

Journal of

Function Spaces

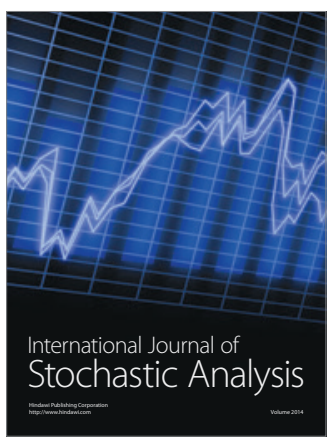

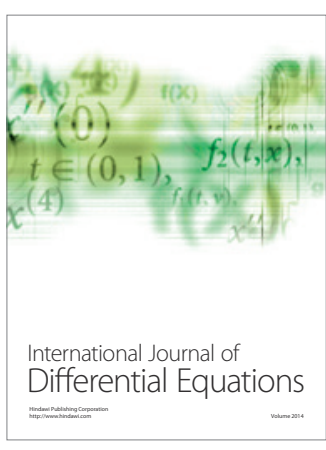
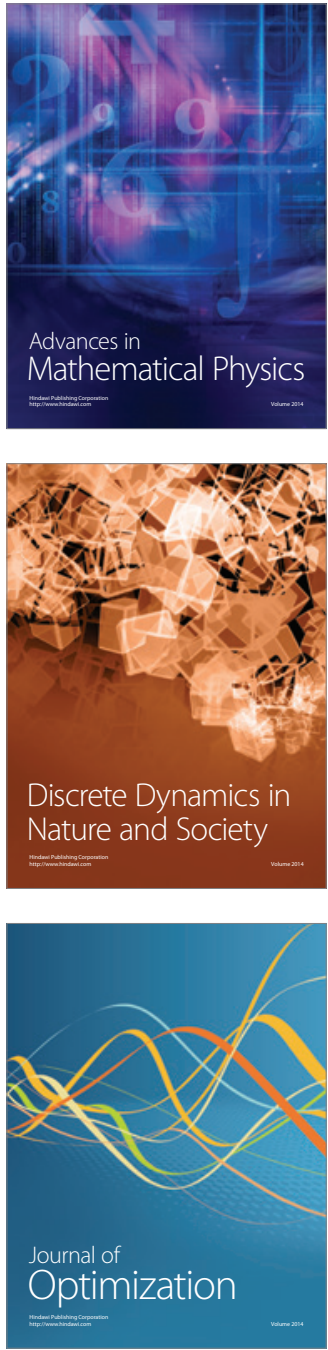